\title{
Output Feedback Receding Horizon Control of Constrained Systems
}

\author{
Paul J. Goulart ${ }^{\dagger, *}$ and Eric C. Kerrigan ${ }^{\ddagger}$ \\ ${ }^{\dagger}$ Department of Engineering, University of Cambridge, Cambridge CB2 1PZ, UK \\ $\ddagger$ Department of Aeronautics and Department of Electrical and Electronic Engineering, \\ Imperial College London, Exhibition Road, London SW7 2AZ, UK
}

(Received 00 Month 200x; In final form 00 Month 200x)

\begin{abstract}
This paper considers output feedback control of linear discrete-time systems with convex state and input constraints which are subject to bounded state disturbances and output measurement errors. We show that the non-convex problem of finding a constraint admissible affine output feedback policy over a finite horizon, to be used in conjunction with a fixed linear state observer, can be converted to an equivalent convex problem. When used in the design of a time-varying robust receding horizon control law, we derive conditions under which the resulting closed-loop system is guaranteed to satisfy the system constraints for all time, given an initial state estimate and bound on the state estimation error. When the state estimation error bound matches the minimal robust positively invariant (mRPI) set for the system error dynamics, we show that this control law is actually time-invariant, but its calculation generally requires solution of an infinite-dimensional optimization problem. Finally, using an invariant outer approximation to the mRPI error set, we develop a time-invariant control law that can be computed by solving a finite-dimensional, tractable optimization problem at each time step, which guarantees that the closed-loop system satisfies the constraints for all time.
\end{abstract}

Keywords: Robustness; output feedback; constrained control; predictive control

\section{Introduction}

This paper considers the problem of output feedback control of linear discrete-time systems with mixed state and input constraints, subject to bounded disturbances on the states and measurements. The main aim is to provide a method for efficient calculation of feedback policies that ensure that the state and input constraints are satisfied for all time, while ensuring that the domain of attraction of the resulting closed-loop system is as large as possible.

The problem of formulating robust control policies that guarantee constraint satisfaction is a longstanding one in the control literature $[8,10,45]$, and various methods have been devised for its solution; these include methods based on robust dynamic programming [7], set invariance [11], $\ell_{1}$ control [16], reference governors [18] and predictive control [4, 13, 28].

A variety of techniques have been proposed for the off-line calculation of output feedback control laws which robustly satisfy system constraints for this type of problem. These include methods employing dynamic linear control laws [12, 16, 42], set valued observers coupled with static nonlinear set-valued maps [40, 41], and controllers based on robust dynamic programming [1,32]; these methods typically suffer from very high computational complexity or excessive conservativeness.

Within the predictive control literature, in which a finite-horizon optimal control problem is solved online at each time instant and applied to the plant in receding horizon fashion, techniques for guaranteeing stability and constraint satisfaction for undisturbed systems via state feedback are now well established; see, for example, the excellent surveys in $[17,30,33]$. More problematic has been the development of robust receding horizon control policies (RHC) for uncertain systems, where one wishes to guarantee constraint satisfaction for all possible realizations of the system uncertainty. It is now generally accepted that, in order to provide a reasonable domain of attraction, optimization must be performed over a sequence of feedback

${ }^{*}$ Corresponding author. Email: pjg42@cam.ac.uk 
policies, rather than over fixed input sequences, otherwise problems of infeasibility may quickly arise [4, 30]. Unfortunately, optimization over arbitrary nonlinear feedback policies is generally computationally intractable, leading to optimization problems whose size grows exponentially with the problem data [39, 44].

For robust predictive control using output feedback, a common ad-hoc approach is to employ an observer and substitute the resulting state estimate in place of the true system state in conjunction with a standard predictive control scheme [17, 33]. However, in order to ensure that the region of attraction is as large as possible while guaranteeing robust constraint satisfaction, an explicit model of the estimation error seems necessary, and a number of control schemes based on error set membership estimation [9,38] have been proposed $[3,15]$. When the system dynamics are linear, a common approach is to employ a combination of a fixed linear observer and associated estimation error set with a fixed stabilizing linear control law, to which a sequence of input perturbations is calculated at each time instant. Variations on this theme have been proposed in $[26,37,46]$, and may be considered the output feedback counterparts to the state feedback methods proposed in $[2,14,25]$.

A related technique from the predictive control literature is to define a 'tube' of trajectories based on a controlled invariant set [24], within which the true state of the system is guaranteed to remain, and to treat the problem as one of steering this set to the origin, where the initial reference state (the 'center' of the tube at the initial time) is treated as a decision variable. The invariant set from which the tube is constructed is typically determined off-line by defining a fixed linear feedback law (see [31] for the state feedback and [29] for the output feedback case), though other methods for defining this set are possible [36].

An obvious method for increasing the domain of attraction using these methods is to compute an affine feedback control law on-line at each sample time - a non-convex problem which has until recently been thought to be intractable. However, for the full information case [21, 27] proposed an alternative convex parameterization based on disturbance feedback, which was later shown to be equivalent to one based on affine state feedback in [20]. In the present paper, an analogous reparameterization for output feedback is presented, together with techniques for synthesizing robust time-invariant RHC laws from this parameterization that guarantee constraint satisfaction for all time, and for which the control input at each time instant can be solved via a single, tractable convex optimization problem.

The proposed method has its origins in the recent work on robust optimization of [6, 21], who developed a novel method for the solution of adjustable robust counterpart (ARC) optimization problems, in which a subset of the decision variables may be selected after some or all of the uncertain problem parameters are realized. It was shown that if these decision parameters are restricted to be affine functions of the system uncertainty, rather than arbitrary nonlinear functions, then the resulting optimization problem is convex and tractable under certain conditions.

The convex control parameterization presented here was originally proposed for robust control of linear systems in $[5,43]$, but without the inclusion of non-zero initial conditions or oberver dynamics. We employ the parameterization in conjunction with a fixed linear state observer and a corresponding bound on the state estimation error, and show that RHC laws synthesized from the parameterization can guarantee constraint satisfaction for all time. When the state estimation error bound matches the minimal robust positively invariant (mRPI) set for the system error dynamics, we show that the control law is actually time-invariant, but its calculation requires the solution of an infinite-dimensional optimization problem when the mRPI set is not finitely determined. Finally, by employing an invariant outer approximation to the mRPI error set [35], we develop a time-invariant control law that can be computed by solving a finite-dimensional tractable optimization problem at each time step.

The paper is organized as follows. Section 2 discusses the class of systems considered and defines a number of standing assumptions. Section 3 defines the affine output feedback policies considered throughout, and, in a manner similar to [5] but taking explicit account of the state estimate and observer error dynamics, demonstrates that one can define an equivalent but convex reparameterization based on output error feedback. This equivalence is then exploited in Section 4 which develops results on invariance and constraint satisfaction of RHC laws synthesized from this parameterization, and which contains the main contributions of the paper. Section 5 demonstrates how the proposed control law may be implemented via the solution of a single linear program (LP) at each time when all of the relevant constraints are polytopic, and provides a short numerical example. Some concluding remarks are made in Section 6. 
Notation: $\mathcal{B}_{p}^{n}(r):=\left\{x \in \mathbb{R}^{n} \mid\|x\|_{p} \leq r\right\}$ is the $p$-norm ball in $\mathbb{R}^{n}$, where $r \geq 0$. Given sets $X \subset \mathbb{R}^{n}$ and $Y \subset \mathbb{R}^{n}$, the Minkowski sum is defined as $X \oplus Y:=\{x+y \mid x \in X, y \in Y\}$. Given a sequence of sets $\left\{X_{i} \subset \mathbb{R}^{n}\right\}_{i=a}^{b}$, define $\oplus_{i=a}^{b} X_{i}:=X_{a} \oplus \cdots \oplus X_{b}$.

\section{Problem Description}

Throughout, we consider the following discrete-time linear time-invariant system:

$$
\begin{aligned}
x^{+} & =A x+B u+w \\
y & =C x+\eta
\end{aligned}
$$

where $x \in \mathbb{R}^{n}$ is the system state at the current time instant, $x^{+}$is the state at the next time instant, $u \in \mathbb{R}^{m}$ is the system input, $w \in \mathbb{R}^{n}$ is a disturbance, $y \in \mathbb{R}^{q}$ is the system output and $\eta \in \mathbb{R}^{q}$ is the measurement error. We assume that the pairs $(A, B)$ and $(C, A)$ are stabilizable and detectable respectively, and that there exist a controller gain $K$ and Luenberger type observer gain $L$ such that the matrices $A_{K}:=(A+B K)$ and $A_{L}:=(A-L C)$ are strictly stable. We define the estimated state $s \in \mathbb{R}^{n}$ at the current time instant such that

$$
s^{+}=A s+B u+L(y-C s)
$$

and define the state estimation error $e \in \mathbb{R}^{n}$ as $e:=x-s$, such that

$$
e^{+}=(A-L C) e-L \eta+w,
$$

where $s^{+}$and $e^{+}$represent the state estimate and estimation error at the next time instant. The actual values of the state, state estimate, estimation error, input and output at time instant $k$ are denoted $x(k)$, $s(k), e(k), u(k)$ and $y(k)$, respectively. We define $\mathrm{E}$ to be the set of all convex and compact subsets of $\mathbb{R}^{n}$ containing the origin, and will typically specify that the estimation error $e$ and true initial state $x$ are such that $e \in \mathcal{E}$ and $x \in\{s\} \oplus \mathcal{E}$, for some given $\mathcal{E} \in \mathrm{E}$. We assume that the system is subject to mixed constraints on the states and inputs, so that the system must satisfy

$$
(x, u) \in Z,
$$

where $Z \subset \mathbb{R}^{n} \times \mathbb{R}^{m}$ is a closed and convex set containing the origin in its interior, and note that such a constraint may include constraints on the output $y$ in (2). We further define a closed and convex target/terminal set $X_{f} \subset \mathbb{R}^{n} \times \mathbb{R}^{n}$ for the state estimate and error, such that $(s, e) \in X_{f}$. We assume that the disturbances $w$ are unknown but contained in a compact set containing the origin in its interior. We denote the set of all such subsets of $\mathbb{R}^{n}$ as $\mathbf{W}$, and will typically specify that $w \in W$ for some $W \in \mathrm{W}$. We further assume the measurement errors $\eta$ are unknown but contained in a compact set $H$, also containing the origin.

Before proceeding, we define some additional notation. In the sequel, predictions of the system's evolution over a finite control/planning horizon will be used to define a number of suitable control policies. Let the length $N$ of this planning horizon be a positive integer and define stacked versions of the state estimate, estimation error, input, output, disturbance, and measurement error vectors $\mathbf{s} \in \mathbb{R}^{n(N+1)}$, e $\in \mathbb{R}^{n(N+1)}$, 
$\mathbf{u} \in \mathbb{R}^{m N}, \mathbf{y} \in \mathbb{R}^{q N}, \mathbf{w} \in \mathbb{R}^{n N}$, and $\boldsymbol{\eta} \in \mathbb{R}^{q N}$ respectively, as

$$
\begin{aligned}
\mathbf{s} & :=\left[s_{0}^{\prime}, \ldots, s_{N}^{\prime}\right]^{\prime}, & \mathbf{e} & :=\left[e_{0}^{\prime}, \ldots, e_{N}^{\prime}\right]^{\prime}, \\
\mathbf{u} & :=\left[u_{0}^{\prime}, \ldots, u_{N-1}^{\prime}\right]^{\prime}, & \mathbf{y} & :=\left[y_{0}^{\prime}, \ldots, y_{N-1}^{\prime}\right]^{\prime}, \\
\mathbf{w} & :=\left[w_{0}^{\prime}, \ldots, w_{N-1}^{\prime}\right]^{\prime}, & \boldsymbol{\eta} & :=\left[\eta_{0}^{\prime}, \ldots, \eta_{N-1}^{\prime}\right]^{\prime},
\end{aligned}
$$

where $s_{0}:=s$ and $e_{0}:=e$ denote the current values of the state estimate and estimation error respectively, and $s_{i+1}:=A_{L} s_{i}+B u_{i}+L y_{i}$ and $e_{i+1}=A_{L} e_{i}-L \eta_{i}+w_{i}, \forall i \in\{0, \ldots, N-1\}$, denote the predictions of the state estimate and estimation error after $i$ time instants. The predicted measurements after $i$ time instants are $y_{i}=C\left(s_{i}+e_{i}\right)+\eta_{i}, \forall i \in\{0, \ldots, N-1\}$.

We define a closed and convex set $\mathcal{Z}$, appropriately constructed from $Z$ and $X_{f}$, such that the constraints to be satisfied are equivalent to $(\mathbf{s}, \mathbf{e}, \mathbf{u}) \in \mathcal{Z}$, i.e.

$$
\mathcal{Z}:=\left\{\begin{array}{l|c}
(\mathbf{s}, \mathbf{e}, \mathbf{u}) & \begin{array}{c}
\left(s_{i}+e_{i}, u_{i}\right) \in Z, \forall i \in\{0, \ldots, N-1\} \\
\left(s_{N}, e_{N}\right) \in X_{f}
\end{array}
\end{array}\right\}
$$

Finally, we define the matrices $\mathbf{A}, \mathbf{B}, \mathbf{C}, \mathbf{E}, \mathcal{L}, \mathcal{B}, \Phi$ and $\Gamma$ (given in the Appendix) and affine functions $f_{e}$ and $f_{s}$ such that the vectors $\mathbf{s}$ and $\mathbf{e}$ can be written as

$$
\begin{aligned}
& \mathbf{s}=f_{s}\left(s_{0}, e_{0}, \mathbf{u}, \mathbf{w}, \boldsymbol{\eta}\right):=\mathbf{A} s_{0}+\mathbf{B u}+\mathbf{E} \mathcal{L}(\mathbf{C e}+\boldsymbol{\eta}) \\
& \mathbf{e}=f_{e}\left(e_{0}, \mathbf{w}, \boldsymbol{\eta}\right) \quad:=\Phi e_{0}-\Gamma \mathcal{L} \boldsymbol{\eta}+\Gamma \mathbf{w}
\end{aligned}
$$

such that $\mathbf{s}$ may alternatively be expressed directly as an affine function of $\mathbf{y}$, i.e.

$$
\mathbf{s}=\Phi s_{0}+\Gamma \mathcal{B} \mathbf{u}+\Gamma \mathcal{L} \mathbf{y}
$$

\section{Affine Feedback Parameterizations}

\subsection{Output Feedback}

As a means of controlling the system (1) while ensuring the satisfaction of the constraints (7) for all possible realizations of the system uncertainty, we wish to construct a control policy such that each control input $u_{i}$ is affine in the measurements $\left\{y_{0}, \ldots, y_{i-1}\right\}$, i.e.

$$
u_{i}=g_{i}+\sum_{j=0}^{i-1} K_{i, j} y_{j}
$$

where each $K_{i, j} \in \mathbb{R}^{m \times p}$ and $g_{i} \in \mathbb{R}^{m}$. For notational convenience we define the vector $\mathbf{g} \in \mathbb{R}^{m N}$ and $\operatorname{matrix} \mathbf{K} \in \mathbb{R}^{m N \times q N}$ as

$$
\mathbf{K}:=\left[\begin{array}{cccc}
0 & \cdots & \cdots & 0 \\
K_{1,0} & \ddots & \cdots & 0 \\
\vdots & \ddots & \ddots & \vdots \\
K_{N-1,0} & \cdots & K_{N-1, N-2} & 0
\end{array}\right], \mathbf{g}:=\left[\begin{array}{c}
g_{0} \\
g_{1} \\
\vdots \\
g_{N-1}
\end{array}\right]
$$

so that the control input sequence can be written as $\mathbf{u}=\mathbf{K} \mathbf{y}+\mathbf{g}$.

For a given initial state estimate $s$, estimation error set $\mathcal{E} \in \mathrm{E}$ and disturbance set $W \in \mathrm{W}$, the set of feasible output feedback policies which are guaranteed to satisfy the constraints $\mathcal{Z}$ for all possible 
uncertainty realizations (assuming that the true initial state $x \in\{s\} \oplus \mathcal{E}$ ) is

$$
\Pi_{N}^{o f}(s, \mathcal{E}, W):=\left\{(\mathbf{K}, \mathbf{g}) \mid \begin{array}{r}
(\mathbf{K}, \mathbf{g}) \text { satisfies }(12) \\
\mathbf{s}=f_{s}(s, e, \mathbf{u}, \mathbf{w}, \boldsymbol{\eta}) \\
\mathbf{e}=f_{e}(e, \mathbf{w}, \boldsymbol{\eta}) \\
\mathbf{y}=\mathbf{C}(\mathbf{s}+\mathbf{e})+\boldsymbol{\eta} \\
\mathbf{u}=\mathbf{K y}+\mathbf{g},(\mathbf{s}, \mathbf{e}, \mathbf{u}) \in \mathcal{Z} \\
\forall e \in \mathcal{E}, \forall \mathbf{w} \in W^{N}, \forall \boldsymbol{\eta} \in H^{N}
\end{array}\right\}
$$

or, equivalently,

$$
\left.\Pi_{N}^{o f}(s, \mathcal{E}, W)=\bigcap_{\substack{\mathbf{w} \in W^{N} \\
\boldsymbol{\eta} \in H^{N}, e \in \mathcal{E}}}\{\mathbf{K}, \mathbf{g}) \mid \begin{array}{r}
(\mathbf{K}, \mathbf{g}) \text { satisfies }(12) \\
\mathbf{s}=f_{s}(s, e, \mathbf{u}, \mathbf{w}, \boldsymbol{\eta}) \\
\mathbf{e}=f_{e}(e, \mathbf{w}, \boldsymbol{\eta}) \\
\mathbf{y}=\mathbf{C}(\mathbf{s}+\mathbf{e})+\boldsymbol{\eta} \\
\mathbf{u}=\mathbf{K} \mathbf{y}+\mathbf{g},(\mathbf{s}, \mathbf{e}, \mathbf{u}) \in \mathcal{Z}
\end{array}\right\}
$$

For a given estimation error set $\mathcal{E} \in \mathrm{E}$ and disturbance set $W \in \mathrm{W}$, we define the set of all initial state estimates for which a constraint admissible policy exists as

$$
\mathcal{S}_{N}^{o f}(\mathcal{E}, W):=\left\{s \mid \Pi_{N}^{o f}(s, \mathcal{E}, W) \neq \emptyset\right\}
$$

Remark 1 The feedback policy (11) includes the class of "pre-stabilizing" control policies in which the control is based on perturbations $\left\{c_{i}\right\}_{i=0}^{N-1}$ to a fixed linear state feedback gain $K$, so that $u_{i}=K s_{i}+c_{i}$, since the estimated state $s_{i}$ may be expressed as an affine function of the measurements $\left\{y_{0}, \ldots, y_{i-1}\right\}$ (cf. (10)). Such a scheme is commonly employed for robust control of constrained systems under state feedback $[2,14,25]$, or employed in conjunction with a stabilizing linear observer gain $L$ for output feedback $[26,37,46]$. The method we propose can also be shown to subsume tube-based schemes such as $[29,31]$ when the invariant sets defining the tube are based on static linear feedback, though these methods also confer additional stability properties which we do not address here.

Remark 2 As in the full state information case considered in [20], the set $\Pi_{N}^{o f}(s, \mathcal{E}, W)$ is non-convex, in general, due to the nonlinear relationship between the estimated states $\mathbf{s}$ and feedback gains $\mathbf{K}$ in (13).

\subsection{Output Error Feedback}

As an alternative to the parameterization (11), we consider a control policy parameterized as an affine function of the uncertain parameters $\mathbf{w}, \boldsymbol{\eta}$ and $e$; a related parameterization was first suggested as a means for finding solutions to a general class of robust optimization problems, called affinely adjustable robust counterpart (AARC) problems [6, 21], and recently as a means for robust control of systems with full state feedback $[20,27]$ and output feedback $[5,43]$. The control policy is parameterized as

$$
u_{i}=v_{i}+\sum_{j=0}^{i-1} M_{i, j}\left(y_{j}-C s_{j}\right)
$$


where each $M_{i, j} \in \mathbb{R}^{m \times p}$ and $v_{i} \in \mathbb{R}^{m}$, and note that $\left(y_{i}-C s_{i}\right)=\left(C e_{i}+\eta_{i}\right)$ for all $i \in\{0, \ldots, N-1\}$. We further define matrices $\mathbf{M} \in \mathbb{R}^{m N \times n N}$ and vector $\mathbf{v} \in \mathbb{R}^{m N}$ as

$$
\mathbf{M}:=\left[\begin{array}{cccc}
0 & \cdots & \cdots & 0 \\
M_{1,0} & 0 & \cdots & 0 \\
\vdots & \ddots & \ddots & \vdots \\
M_{N-1,0} & \cdots & M_{N-1, N-2} & 0
\end{array}\right], \mathbf{v}:=\left[\begin{array}{c}
v_{0} \\
v_{1} \\
\vdots \\
v_{N-1}
\end{array}\right]
$$

so that the control input sequence can be written as

$$
\begin{aligned}
\mathbf{u} & =\mathbf{M}(\mathbf{y}-\mathbf{C s})+\mathbf{v} \\
& =\mathbf{M}(\mathbf{C e}+\boldsymbol{\eta})+\mathbf{v}
\end{aligned}
$$

By virtue of the relation (9), this control parameterization is affine in the unknown parameters $e$, w, $\boldsymbol{\eta}$. For a given initial state estimate $s$, estimation error set $\mathcal{E} \in \mathrm{E}$ and disturbance set $W \in \mathrm{W}$, the set of feasible feedback policies that are guaranteed to satisfy the system constraints for all possible uncertainty realizations (assuming that the true initial state $x \in\{s\} \oplus \mathcal{E}$ ) is

$$
\Pi_{N}^{e f}(s, \mathcal{E}, W):=\left\{(\mathbf{M}, \mathbf{v}) \mid \begin{array}{r}
(\mathbf{M}, \mathbf{v}) \text { satisfies }(16) \\
\mathbf{s}=f_{s}(s, e, \mathbf{u}, \mathbf{w}, \boldsymbol{\eta}) \\
\mathbf{e}=f_{e}(e, \mathbf{w}, \boldsymbol{\eta}) \\
\mathbf{y}=\mathbf{C}(\mathbf{s}+\mathbf{e})+\boldsymbol{\eta} \\
\\
\mathbf{u}=\mathbf{M}(\mathbf{y}-\mathbf{C s})+\mathbf{v},(\mathbf{s}, \mathbf{e}, \mathbf{u}) \in \mathcal{Z} \\
\forall e \in \mathcal{E}, \forall \mathbf{w} \in W^{N}, \forall \boldsymbol{\eta} \in H^{N}
\end{array}\right\}
$$

or, equivalently,

$$
\Pi_{N}^{e f}(s, \mathcal{E}, W)=\bigcap_{\substack{\mathbf{w} \in W^{N} \\
\boldsymbol{\eta} \in H^{N}, e \in \mathcal{E}}}\left\{\begin{array}{r}
(\mathbf{M}, \mathbf{v}) \text { satisfies }(16) \\
\mathbf{s}=f_{s}(s, e, \mathbf{u}, \mathbf{w}, \boldsymbol{\eta}) \\
\mathbf{e}=f_{e}(e, \mathbf{w}, \boldsymbol{\eta}) \\
\mathbf{y}=\mathbf{C}(\mathbf{s}+\mathbf{e})+\boldsymbol{\eta} \\
\\
\mathbf{u}=\mathbf{M}(\mathbf{C e}+\boldsymbol{\eta})+\mathbf{v},(\mathbf{s}, \mathbf{e}, \mathbf{u}) \in \mathcal{Z}
\end{array}\right\}
$$

For a given error set $\mathcal{E} \in \mathrm{E}$ and disturbance set $W \in \mathrm{W}$, define the set of all constraint admissible initial state estimates to be

$$
\mathcal{S}_{N}^{e f}(\mathcal{E}, W):=\left\{s \mid \Pi_{N}^{e f}(s, \mathcal{E}, W) \neq \emptyset\right\}
$$

We next characterize two critical properties of the parameterization (15), which make it attractive in application to control of the system (1), and which parallel the results in [20] for the full state feedback case.

\subsubsection{Convexity.}

TheOREM 3.1 For any $\mathcal{E} \in \mathrm{E}$ and any $W \in \mathrm{W}$, the set of constraint admissible feedback policies $\Pi_{N}^{e f}(s, \mathcal{E}, W)$ is convex and closed, and the set of feasible initial states $\mathcal{S}_{N}^{e f}(\mathcal{E}, W)$ is convex. 
Proof Define the set

$$
\mathcal{C}_{N}(\mathcal{E}, W):=\bigcap_{\substack{\mathbf{w} \in W^{N} \\
\boldsymbol{\eta} \in H^{N}, e \in \mathcal{E}}}\left\{\begin{array}{r}
(\mathbf{M}, \mathbf{v}, s) \mid \begin{array}{r}
(\mathbf{M}, \mathbf{v}) \text { satisfies }(16) \\
\mathbf{s}=f_{s}(s, e, \mathbf{u}, \mathbf{w}, \boldsymbol{\eta}) \\
\mathbf{e}=f_{e}(e, \mathbf{w}, \boldsymbol{\eta}) \\
\mathbf{y}=\mathbf{C}(\mathbf{s}+\mathbf{e})+\boldsymbol{\eta} \\
\mathbf{u}=\mathbf{M}(\mathbf{C e}+\boldsymbol{\eta})+\mathbf{v},(\mathbf{s}, \mathbf{e}, \mathbf{u}) \in \mathcal{Z}
\end{array}
\end{array}\right\}
$$

which is closed and convex, since it is the intersection of closed and convex sets. The set $\mathcal{S}_{N}^{e f}(\mathcal{E}, W)$ is a projection of this set, and is thus convex. The set $\Pi_{N}^{e f}(s, \mathcal{E}, W)$ is similarly written as an intersection of closed and convex sets, so is also closed and convex.

Remark 3 In certain cases it is possible to find a feasible policy $(\mathbf{M}, \mathbf{v}) \in \Pi_{N}^{e f}(s, \mathcal{E}, W)$ given an initial state estimate $s$ using standard techniques in convex optimization similar to those required in the case of robust control with state feedback [20]. For example, if the constraint set $\mathcal{Z}$ and uncertainty sets $W, H$ and $\mathcal{E}$ are polytopes, a constraint admissible policy $(\mathbf{M}, \mathbf{v}) \in \Pi_{N}^{e f}(s, \mathcal{E}, W)$ can be found by solving a single, tractable linear program (LP). If $\mathcal{Z}$ is a polytope and the sets $\mathcal{E}, H$ and $W$ are ellipsoids, then a constraint admissible policy can be found by solving a single, tractable second-order cone program (SOCP).

\subsubsection{Equivalence.}

TheOrem 3.2 Given any initial state estimation set $\mathcal{E} \in \mathrm{E}$ and any disturbance set $W \in \mathrm{W}$, the sets $\mathcal{S}_{N}^{e f}(\mathcal{E}, W)$ and $\mathcal{S}_{N}^{o f}(\mathcal{E}, W)$ are equal. For a given state estimate $s$, for every pair $(\mathbf{K}, \mathbf{g}) \in \Pi_{N}^{o f}(s, \mathcal{E}, W)$, there exists a pair $(\mathbf{M}, \mathbf{v}) \in \Pi_{N}^{e f}(s, \mathcal{E}, W)$ giving the same sequence of inputs and states for all possible realizations of the system uncertainty, and vice-versa.

Proof $\mathcal{S}_{N}^{o f}(\mathcal{E}, W) \subseteq \mathcal{S}_{N}^{e f}(\mathcal{E}, W)$ : By definition, for any $s \in \mathcal{S}_{N}^{o f}(\mathcal{E}, W)$, there exists a pair $(\mathbf{K}, \mathbf{g}) \in$ $\Pi_{N}^{e f}(s, \mathcal{E}, W)$. A bit of algebra shows that, given any uncertainty realization $e, \boldsymbol{\eta}$ and $\mathbf{w}$, the input sequence $\mathbf{u}$ can be written as

$$
\mathbf{u}=\Delta^{-1} \mathbf{K}[\mathbf{C}(\mathbf{A} e+\mathbf{E w})+\boldsymbol{\eta}]+\Delta^{-1}(\mathbf{K C A} s+g),
$$

where $\Delta:=(I-\mathbf{K C B})$, and the matrix $\Delta$ is always invertible since $\mathbf{K C B}$ is strictly lower triangular. Noting the identity $\mathbf{C}(\mathbf{A} e+\mathbf{E w})+\boldsymbol{\eta}=(I+\mathbf{C E} \mathcal{L})(\mathbf{y}-\mathbf{C} s)$, the input sequence $\mathbf{u}$ can thus be written as

$$
\mathbf{u}=\Delta^{-1} \mathbf{K}(I+\mathbf{C E} \mathcal{L})(\mathbf{y}-\mathbf{C} s)+\Delta^{-1}(\mathbf{K C A} s+g)
$$

A constraint admissible policy $(\mathbf{M}, \mathbf{v}) \in \mathcal{S}_{N}^{e f}(\mathcal{E}, W)$ can then be found by selecting

$$
\mathbf{M}=\Delta^{-1} \mathbf{K}(I+\mathbf{C E} \mathcal{L}), \quad \mathbf{v}=\Delta^{-1}(\mathbf{K C A} s+g) .
$$

Thus, $s \in \mathcal{S}_{N}^{e f}(\mathcal{E}, W)$ for all $s \in \mathcal{S}_{N}^{o f}(\mathcal{E}, W)$, so $\mathcal{S}_{N}^{o f}(\mathcal{E}, W) \subseteq \mathcal{S}_{N}^{e f}(\mathcal{E}, W)$.

$\mathcal{S}_{N}^{e f}(\mathcal{E}, W) \subseteq \mathcal{S}_{N}^{o f}(\mathcal{E}, W)$ : By definition, for any $s \in \mathcal{S}_{N}^{e f}(\mathcal{E}, W)$, there exists a pair $(\mathbf{M}, \mathbf{v}) \in \Pi_{N}^{o f}(s, \mathcal{E}, W)$. Using the relation (10), the output error terms can be written as $\mathbf{y}-\mathbf{C s}=(I-\mathbf{C} \Gamma \mathcal{L}) \mathbf{y}-C \Phi s-C \Gamma \mathcal{B} \mathbf{u}$, and the control input sequence $\mathbf{u}=\mathbf{M}(\mathbf{y}-\mathbf{C s})+\mathbf{v}$ as

$$
\mathbf{u}=\hat{\Delta}^{-1} \mathbf{M}(I-\mathbf{C} \Gamma \mathcal{L}) \mathbf{y}+\hat{\Delta}^{-1}(\mathbf{v}-\mathbf{M C} \Phi s)
$$

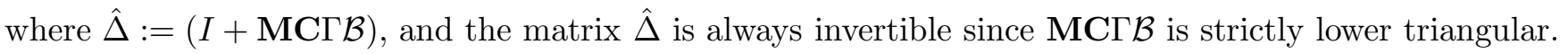


A constraint admissible policy $(\mathbf{K}, \mathbf{g}) \in \mathcal{S}_{N}^{o f}(\mathcal{E}, W)$ can then be found by selecting

$$
\mathbf{K}=\hat{\Delta}^{-1} \mathbf{M}(I-\mathbf{C} \Gamma \mathcal{L}), \mathbf{g}=\hat{\Delta}^{-1}(\mathbf{v}-\mathbf{M C} \Phi s)
$$

Thus, $s \in \mathcal{S}_{N}^{o f}(\mathcal{E}, W)$ for all $s \in \mathcal{S}_{N}^{e f}(\mathcal{E}, W)$, so $\mathcal{S}_{N}^{e f}(\mathcal{E}, W) \subseteq \mathcal{S}_{N}^{o f}(\mathcal{E}, W)$.

Remark 4 A control policy based on the measurement prediction error terms ( $\mathbf{y}-\mathbf{C s})$ was proposed in [43], and independently in the context of robust optimization in [5]. The latter gives an equivalence proof similar to that presented here, but without the inclusion of a non-zero initial state estimate or observer dynamics. In the sequel, we make explicit use of these error dynamics to derive conditions under which receding horizon control (RHC) laws based on the parameterization (15) can be guaranteed to satisfy constraints for the resulting closed-loop system for all time.

\section{Geometric and Invariance Properties}

In this section, we characterize some of the geometric and invariance properties associated with control laws synthesized from the feedback parameterization (15). We first require the following assumption about the terminal constraint set $X_{f}$ :

A1 (Terminal constraint) For a given disturbance set $W \in \mathrm{W}$, the state feedback gain matrix $K$, observer gain $L$ and terminal constraint set $X_{f}$ have been chosen such that:

- $X_{f}$ is consistent with the set of states for which the constraints $Z$ in (5) are satisfied under the control $u=K s$, i.e. $(s, e) \in X_{f}$ implies $(s+e, K s) \in Z$.

- $X_{f}$ is robust positively invariant for the closed-loop system under the control $u=K s$. Thus $(s, e) \in X_{f}$ guarantees $\left(s^{+}, e^{+}\right) \in X_{f}$ for all $w \in W$ and for all $\eta \in H$, where $s^{+}=(A+B K) s+L(C e+\eta)$ and $e^{+}=A_{L} e-L \eta+w$.

Remark 1 If the set $W \times H$ is a polytope or affine map of a $p$-norm ball and the constraints $Z$ are polyhedral, then one can calculate an invariant set which satisfies the conditions $\mathbf{A} \mathbf{1}$ by applying the techniques in $[11,23]$ to the augmented system

$$
\left[\begin{array}{l}
s^{+} \\
e^{+}
\end{array}\right]=\left[\begin{array}{cc}
(A+B K) & L C \\
0 & (A-L C)
\end{array}\right]\left[\begin{array}{l}
s \\
e
\end{array}\right]+\left[\begin{array}{cc}
0 & L \\
I-L
\end{array}\right]\left[\begin{array}{l}
w \\
\eta
\end{array}\right]
$$

In general, one wishes to select the terminal set $X_{f}$ such that is a maximal invariant set, so that the the set $\Pi_{N}(\mathcal{E}, W)$ is as large as possible. Alternatively, one may can use the techniques in [25] for calculating a target set of a given complexity.

Proposition 4.1 (Monotonicity) If $\boldsymbol{A} \mathbf{1}$ holds, then the following set inclusions hold for any $\mathcal{E} \in \mathrm{E}$ and any $W \in \mathrm{W}$ :

$$
\begin{aligned}
& \mathcal{S}_{1}^{o f}(\mathcal{E}, W) \subseteq \cdots \subseteq \mathcal{S}_{N-1}^{o f}(\mathcal{E}, W) \subseteq \mathcal{S}_{N}^{o f}(\mathcal{E}, W) \subseteq \mathcal{S}_{N+1}^{o f}(\mathcal{E}, W) \ldots \\
& \mathcal{S}_{1}^{e f}(\mathcal{E}, W) \subseteq \cdots \subseteq \mathcal{S}_{N-1}^{e f}(\mathcal{E}, W) \subseteq \mathcal{S}_{N}^{e f}(\mathcal{E}, W) \subseteq \mathcal{S}_{N+1}^{e f}(\mathcal{E}, W) \ldots
\end{aligned}
$$

Proof The proof of the first relation is by induction. Suppose that $s \in \mathcal{S}_{N}^{o f}(\mathcal{E}, W)$ and $(\mathbf{K}, \mathbf{g}) \in \Pi_{N}^{o f}(s, \mathcal{E}, W)$. Recalling the relation (10), the state estimates $\mathbf{s}$ can be found as an affine function of the measurements $\mathbf{y}$ using

$$
\mathbf{s}=\Gamma(\mathcal{B K}+\mathcal{L}) \mathbf{y}+\Gamma \mathcal{B} \mathbf{g}+\Phi s_{0}
$$


One can thus find a pair $(\overline{\mathbf{K}}, \overline{\mathbf{g}}) \in \Pi_{N+1}^{o f}(s, \mathcal{E}, W)$, where $\overline{\mathbf{K}}:=\left[\begin{array}{cc}\mathbf{K} & 0 \\ \bar{K}_{1} & \bar{K}_{2}\end{array}\right]$ and $\overline{\mathbf{g}}:=\left[\begin{array}{l}\mathbf{g} \\ \bar{g}\end{array}\right]$, by defining the matrices $\bar{K}_{1}:=\mathcal{A}_{L}(\mathcal{B} \mathbf{K}+\mathcal{L})$ and $\bar{K}_{2}:=0$ and vector $\bar{g}:=\left(\mathcal{A}_{L}(\mathcal{B} \mathbf{K}+\mathcal{L}) \mathbf{g}+A_{L}^{N} s_{0}\right)$, where $\mathcal{A}_{L}:=$ $\left[\begin{array}{lll}A_{L}^{N-1} \cdots & A_{L} I\end{array}\right]$, such that the final stage input is $u_{N}=K s_{N}$. Since $s \in \mathcal{S}_{N}^{o f}(\mathcal{E}, W)$ implies $\left(s_{N}, e_{N}\right) \in X_{f}$ by definition, then it follows that $\left(s_{N}+e_{N}, u_{N}\right) \in Z$ and $\left(s_{N+1}, e_{N+1}\right) \in X_{f}$ for all $w \in W$ and all $\eta \in H$ if $\mathbf{A} 1$ holds. Thus $(\overline{\mathbf{K}}, \overline{\mathbf{g}}) \in \Pi_{N+1}^{o f}(s, \mathcal{E}, W)$ and $s \in \mathcal{S}_{N+1}^{o f}(\mathcal{E}, W)$. The second relation then follows from Theorem 3.2.

\subsection{Invariance Properties}

We next consider some properties of receding horizon control (RHC) laws synthesized from the parameterization (11) (equivalently, (15)). In particular, we develop conditions under which such a RHC law can be guaranteed to be robust positively invariant for the resulting closed-loop system.

We define the set-valued map $\kappa_{N}: \mathbb{R}^{n} \times \mathrm{E} \times \mathrm{W} \rightarrow 2^{\mathbb{R}^{m}}$ as

$$
\begin{aligned}
\kappa_{N}(s, \mathcal{E}, W) & :=\left\{u \mid \exists(\mathbf{K}, \mathbf{g}) \in \Pi_{N}^{o f}(s, \mathcal{E}, W) \text { s.t. } u=g_{0}\right\} \\
& =\left\{u \mid \exists(\mathbf{M}, \mathbf{v}) \in \Pi_{N}^{e f}(s, \mathcal{E}, W) \text { s.t. } u=v_{0}\right\}
\end{aligned}
$$

where $2^{\mathbb{R}^{m}}$ is the set of all subsets of $\mathbb{R}^{m}$, and (30) follows directly from Theorem 3.2. We define a function $\mu_{N}: \mathbb{R}^{n} \times \mathrm{E} \times \mathrm{W} \rightarrow \mathbb{R}^{m}$ as any selection from the set $\kappa_{N}$, i.e. given $\mathcal{E} \in \mathrm{E}$ and $W \in \mathrm{W}, \mu_{N}(\cdot, \mathcal{E}, W)$ must satisfy

$$
\mu_{N}(s, \mathcal{E}, W) \in \kappa_{N}(s, \mathcal{E}, W), \forall s \in \mathcal{S}_{N}^{o f}(\mathcal{E}, W)
$$

We wish to develop conditions under which time-varying or time-invariant control schemes based on the functions $\mu_{N}$ can be guaranteed to satisfy the system constraints $Z$ for all time. We first introduce the following standard definition from the theory of invariant sets [23, 35]:

Definition 4.2 The set $\mathcal{E}_{i}$ is defined as

$$
\mathcal{E}_{i}:=\bigoplus_{j=0}^{i} A_{L}^{j}(W \oplus L(-H)), \quad \forall i \in\{0,1, \ldots\}
$$

The minimal robust positively invariant (mRPI) set $\mathcal{E}_{\infty}$ is defined as the limit set of the sequence $\left\{\mathcal{E}_{i}\right\}$, i.e. $\mathcal{E}_{\infty}:=\lim _{i \rightarrow \infty} \mathcal{E}_{i}$.

Remark 2 As noted in [23], unless the observer gain $L$ is selected such that there exists an integer $k \geq 0$ and $0 \leq \alpha<1$ such that $A_{L}^{k}=\alpha A_{L}$ (e.g. when $L$ is a deadbeat observer so that $A_{L}$ is nilpotent), then the set $\mathcal{E}_{\infty}$ may not be characterized by a finite number of inequalities, since it is a Minkowski sum with an infinite number of terms. However, in [35] it is shown how one can calculate an arbitrarily close outer approximation $\mathcal{E}_{I}$ to the set $\mathcal{E}_{\infty}$ (which can be represented by a tractable number of inequalities if $W$ and $H$ are polytopes) such that $\mathcal{E}_{\infty} \subseteq \mathcal{E}_{I} \subseteq \mathcal{E}_{\infty} \oplus \mathcal{B}_{p}^{n}(\epsilon)$ and such that the set $\mathcal{E}_{I}$ is robust positively invariant. Further, it is shown in [35] that, if only the support function of the set $\mathcal{E}_{I}$ is required, then calculation of an explicit representation of $\mathcal{E}_{I}$ via Minkowski summation is not necessary, a fact which we exploit in the computational results of Section 5 .

\subsubsection{Time-Varying and mRPI-based RHC Laws.}

We first consider the implementation of a time-varying receding horizon control (RHC) law based on the function $\mu_{N}(\cdot)$. Taking the initial time to be 0 (which is always possible since the system (3)-(4) 
is time-invariant), and given an initial state estimate $s(0)$, initial state estimation error set $\mathcal{E} \in \mathrm{E}$ and disturbance set $W \in \mathrm{W}$, we define the time-varying $\mathrm{RHC}$ control law $\nu: \mathbb{R}^{n} \times \mathbb{N} \times \mathrm{E} \times \mathrm{W} \rightarrow \mathbb{R}^{m}$ as

$$
\nu(s(k), k, \mathcal{E}, W):=\left\{\begin{array}{ll}
\mu_{N}(s(k), \mathcal{E}, W), & \text { if } k=0 \\
\mu_{N}\left(s(k), A_{L}^{k} \mathcal{E} \oplus \mathcal{E}_{k-1}, W\right), & \text { if } k>0
\end{array} .\right.
$$

Note that the error sets required in the calculation of $\nu\left(s(k), k, A_{L}^{k} \mathcal{E} \oplus \mathcal{E}_{k-1}, W\right)$ can be defined recursively, i.e. $A_{L}^{k+1} \mathcal{E} \oplus \mathcal{E}_{k}=A_{L}\left[A_{L}^{k} \mathcal{E} \oplus \mathcal{E}_{k-1}\right] \oplus \mathcal{E}_{0}$, though an explicit calculation of these sets via Minkowski summation is not required (cf. Section 5). The resulting closed-loop system can be written as:

$$
\begin{aligned}
x(k+1) & =A x(k)+B \nu(s(k), k, \mathcal{E}, W)+w \\
s(k+1) & =A s(k)+B \nu(s(k), k, \mathcal{E}, W)+L(y(k)-C s(k)) \\
e(k+1) & =(A-L C) e(k)-L \eta(k)+w(k) \\
y(k) & =C x(k)+\eta(k),
\end{aligned}
$$

where $w \in W$ and $\eta \in H$. Note that given the estimation error set $\mathcal{E}$ at time 0 , the estimation errors $\{e(k)\}_{k=0}^{\infty}$ in (35) are only known by the controller to satisfy $e(k) \in A_{L}^{k} \mathcal{E} \oplus \mathcal{E}_{k-1}$. Our first invariance result follows immediately:

Proposition 4.3 If $\boldsymbol{A} 1$ holds and $s(0) \in \mathcal{S}_{N}^{o f}(\mathcal{E}, W)$, then the closed-loop system (33)-(36) satisfies the constraints (5) for all time and all possible uncertainty realizations if the true initial state $x(0) \in\{s(0)\} \oplus \mathcal{E}$.

Proof If $s \in \mathcal{S}_{N}^{o f}(\tilde{\mathcal{E}}, W)$ for some $\tilde{\mathcal{E}} \in \mathrm{E}$, then there exists an output feedback policy pair $(\mathbf{K}, \mathbf{g}) \in \Pi_{N}^{o f}(s, \tilde{\mathcal{E}}, W)$ for which $\mu_{N}(s, \tilde{\mathcal{E}}, W)=g_{0}$. It is then easy to show that

$$
s^{+}=A s+B \mu_{N}(s, \tilde{\mathcal{E}}, W)+L(C e+\eta) \in \mathcal{S}_{N-1}^{o f}\left(A_{L} \tilde{\mathcal{E}} \oplus \mathcal{E}_{0}, W\right), \forall e \in \tilde{\mathcal{E}}
$$

since one can construct a feasible policy pair $(\tilde{\mathbf{K}}, \tilde{\mathbf{g}}) \in \Pi_{N-1}^{o f}\left(s^{+}, A_{L} \tilde{\mathcal{E}} \oplus \mathcal{E}_{0}, W\right)$ from $(\mathbf{K}, \mathbf{g})$ by dropping the first element of $\mathbf{g}$ and the first block row and column of $\mathbf{K}$. If $\mathbf{A} \mathbf{1}$ holds, then $s^{+} \in \mathcal{S}_{N-1}^{o f}\left(A_{L} \tilde{\mathcal{E}} \oplus \mathcal{E}_{0}, W\right)$ implies $s^{+} \in \mathcal{S}_{N}^{o f}\left(A_{L} \tilde{\mathcal{E}} \oplus \mathcal{E}_{0}, W\right)$ from Proposition 4.1, and the result follows.

We note that if the state estimation error set $\mathcal{E}=\mathcal{E}_{\infty}$, then the control law $\nu(\cdot)$ defined in (32) is actually time-invariant, so that

$$
\nu\left(s(k), k, \mathcal{E}_{\infty}, W\right)=\mu_{N}\left(s(k), \mathcal{E}_{\infty}, W\right), \quad k=0,1, \ldots
$$

The next result follows immediately:

COROLlary 4.4 The set $\mathcal{S}_{N}^{o f}\left(\mathcal{E}_{\infty}, W\right)$ is robust positively invariant for the closed-loop system (33)-(36) under the time-invariant control law (37), i.e. if $s(0) \in \mathcal{S}_{N}^{o f}\left(\mathcal{E}_{\infty}, W\right)$ and $x(0) \in\{s(0)\} \oplus \mathcal{E}_{\infty}$, then $s(k) \in$ $\mathcal{S}_{N}^{o f}\left(\mathcal{E}_{\infty}, W\right)$ and the constraints (5) are satisfied for all time and for all possible uncertainty realizations.

\subsubsection{A Time-Invariant Finite-Dimensional RHC Law.}

The central difficulty with the control law defined in (37) is that, in general, the set $\mathcal{E}_{\infty}$ is not finitely determined (cf. Remark 2). The calculation of the control law $\nu(\cdot, \cdot, \mathcal{E}, W)$ in (32) is thus of increasing complexity with increasing time, and the calculation of the control law $\nu\left(\cdot, \cdot, \mathcal{E}_{\infty}, W\right.$ ) in (37) requires the solution of an infinite-dimensional optimization problem. We thus seek a control law which is of fixed and finite complexity, while preserving the time-invariant nature of (37). To this end, we define a robust positively invariant (RPI) error set $\mathcal{E}_{I} \in \mathrm{E}$ which satisfies the following: 
A2 (Invariant Error Set) For a given disturbance set $W \in \mathrm{W}$, the set $\mathcal{E}_{I} \in \mathrm{E}$ is chosen such that it is robust positively invariant for the system $e^{+}=A_{L} e-L \eta+w$, so that $A_{L} e-L \eta+w \in \mathcal{E}_{I}$ for all $e \in \mathcal{E}_{I}$, $w \in W$ and $\eta \in H$. Furthermore, for some $p$-norm, $\mathcal{E}_{I}$ is an $\epsilon$-outer approximation for $\mathcal{E}_{\infty}$, so that there exists some $\epsilon>0$ such that $\mathcal{E}_{\infty} \subseteq \mathcal{E}_{I} \subseteq \mathcal{E}_{\infty} \oplus \mathcal{B}_{p}^{n}(\epsilon)$.

We can now guarantee an invariance condition similar to the one in Proposition 4.3 using the finitely determined set $\mathcal{E}_{I}$, by slightly enlarging the disturbance set $W$ from which feedback policies of the form (15) are selected. We henceforward assume that the true disturbances are known to be drawn from some set $W \in \mathrm{W}$, and define

$$
W_{\epsilon}:=W \oplus \mathcal{B}_{p}^{n}(\epsilon)
$$

where $p$ and $\epsilon$ satisfy the conditions of $\mathbf{A 2}$ for the set $\mathcal{E}_{I}$. Using this enlarged disturbance set, we consider the following modified assumption on the target/terminal constraint set $X_{f} \subseteq \mathbb{R}^{n} \times \mathbb{R}^{n}$ :

A3 (Modified terminal constraint) The state feedback gain matrix $K$ and modified terminal constraint set $X_{f}$ have been chosen such that:

- $X_{f}$ is consistent with the set of states for which the constraints $Z$ in (5) are satisfied under the control $u=K s$, i.e. $(s, e) \in X_{f}$ implies $(s+e, K s) \in Z$.

- $X_{f}$ is robust positively invariant for the system $s^{+}=(A+B K) s+L(C e+\eta), e^{+}=A_{L} e-L \eta+w$ for all $s \in X_{f}, w \in W \oplus \mathcal{B}_{p}^{n}(\epsilon)$ and $\eta \in H$.

In the sequel, we will choose an invariant set $\mathcal{E}_{I}$ and scalar $\epsilon>0$ satisfying the conditions of $\mathbf{A 2}$ and $\mathbf{A 3}$ such that a time-invariant control law constructed from $\Pi_{N}^{o f}\left(s, \mathcal{E}_{I}, W_{\epsilon}\right)$ (equivalently, $\Pi_{N}^{e f}\left(s, \mathcal{E}_{I}, W_{\epsilon}\right)$ ) can be guaranteed to satisfy the system constraints for all time.

Remark 3 Using the equivalent convex feedback parameterization $(15)$, a pair $(\mathbf{K}, \mathbf{g}) \in \Pi_{N}^{o f}\left(s, \mathcal{E}_{I}, W_{\epsilon}\right)$ can be calculated using standard convex optimization techniques (cf. Remark 3 and Theorem 3.2), where the optimization problem to be solved is finite-dimensional, since the set $\mathcal{E}_{I}$ can be implicitly characterized by a finite number of inequalities (cf. Remark 2). We show in Section 5 that if all of the relevant constraint sets are polytopic, then such a policy can be found via the solution of a single, tractable linear program.

We define the time-invariant control law $\nu_{I}: \mathcal{S}_{N}^{o f}\left(\mathcal{E}_{I}, W_{\epsilon}\right) \rightarrow \mathbb{R}^{m}$ as:

$$
\nu_{I}(s):=\mu_{N}\left(s, \mathcal{E}_{I}, W_{\epsilon}\right) \cdot p
$$

When applied to the control of the system (1), the closed-loop system dynamics become

$$
\begin{aligned}
x^{+} & =A x+B \nu_{I}(s)+w \\
s^{+} & =A s+B \nu_{I}(s)+L(y-C s) . \\
e^{+} & =A_{L} e-L \eta+w \\
y & =C x+\eta,
\end{aligned}
$$

where $w \in W$ and $\eta \in H$. It is critical to note that, though the control law $\nu_{I}(\cdot)$ defined in (39) is conservatively constructed using the enlarged disturbance set $W_{\epsilon}$, the disturbances $w$ in (40) are generated from the true disturbance set $W$. It is this conservativeness which will ensure that the time-invariant control law (39) can guarantee constraint satisfaction of the closed-loop system for all time. We can now state our final result:

Theorem 4.5 If $\boldsymbol{A} \mathscr{2}$ and $\boldsymbol{A} 3$ hold, then the set $\mathcal{S}_{N}^{o f}\left(\mathcal{E}_{I}, W_{\epsilon}\right)$ is robust positively invariant for the closed- 
loop system (40)-(43), i.e. if $s \in \mathcal{S}_{N}^{o f}\left(\mathcal{E}_{I}, W_{\epsilon}\right)$ and $x(0) \in\{s(0)\} \oplus \mathcal{E}_{I}$, then $s^{+} \in \mathcal{S}_{N}^{o f}\left(\mathcal{E}_{I}, W_{\epsilon}\right)$ and the constraints (5) for all time and for all possible uncertainty realizations.

Proof If $\mathbf{A} \mathbf{3}$ holds then it can be shown, using arguments identical to those in the proof of Proposition 4.3, that $s \in \mathcal{S}_{N}^{o f}\left(\mathcal{E}_{I}, W_{\epsilon}\right)$ implies that the successor state $s^{+} \in \mathcal{S}_{N}^{o f}\left(A_{L} \mathcal{E}_{I} \oplus W_{\epsilon} \oplus L(-H), W_{\epsilon}\right)=\mathcal{S}_{N, \epsilon}^{o f}\left(A_{L} \mathcal{E}_{I} \oplus \mathcal{E}_{0} \oplus\right.$ $\left.\mathcal{B}_{p}^{n}(\epsilon), W_{\epsilon}\right)$. If $\mathbf{A} 2$ holds, then $\mathcal{E}_{\infty} \subseteq A_{L} \mathcal{E}_{I} \oplus \mathcal{E}_{0} \subseteq \mathcal{E}_{I}$, and thus $\mathcal{E}_{I} \subseteq \mathcal{E}_{\infty} \oplus \mathcal{B}_{p}^{n}(\epsilon) \subseteq A_{L} \mathcal{E}_{I} \oplus \mathcal{E}_{0} \oplus \mathcal{B}_{p}^{n}(\epsilon)$. Writing the set $\Pi_{N}^{o f}\left(s, \mathcal{E}, W_{\epsilon}\right)$ in terms of set intersections as in (21) it is easy to verify that, for any sets $\mathcal{E}^{\prime} \in \mathrm{E}$ and $\mathcal{E}^{\prime \prime} \in \mathrm{E}, \mathcal{E}^{\prime} \subseteq \mathcal{E}^{\prime \prime}$ implies $\Pi_{N}^{o f}\left(s, \mathcal{E}^{\prime \prime}, W_{\epsilon}\right) \subseteq \Pi_{N}^{o f}\left(s, \mathcal{E}^{\prime}, W_{\epsilon}\right)$ for all $s \in \mathbb{R}^{n}$, and thus $\mathcal{S}_{N}^{o f}\left(\mathcal{E}^{\prime \prime}, W_{\epsilon}\right) \subseteq \mathcal{S}_{N}^{o f}\left(\mathcal{E}^{\prime}, W_{\epsilon}\right)$. It follows that $\mathcal{S}_{N}^{o f}\left(A_{L} \mathcal{E}_{I} \oplus \mathcal{E}_{0} \oplus \mathcal{B}_{p}^{n}(\epsilon), W_{\epsilon}\right) \subseteq \mathcal{S}_{N}^{o f}\left(\mathcal{E}_{I}, W_{\epsilon}\right)$ and thus that $s^{+} \in \mathcal{S}_{N}^{o f}\left(\mathcal{E}_{I}, W_{\epsilon}\right)$ for all $e \in \mathcal{E}_{I}$, $\eta \in H$ and $w \in W$. Finally we verify that the closed-loop system (40)-(43) satisfies the constraints $Z$ for all time; we again use set intersection arguments to confirm that $\Pi_{N}^{o f}\left(s, \mathcal{E}_{I}, W_{\epsilon}\right) \subseteq \Pi_{N}^{o f}\left(s, \mathcal{E}_{I}, W\right)$. This implies that $\kappa_{N}\left(s, \mathcal{E}_{I}, W_{\epsilon}\right) \subseteq \kappa_{N}\left(s, \mathcal{E}_{I}, W\right)$, which guarantees that $\left(s+e, \nu_{I}(s)\right) \in Z$ for all $e \in \mathcal{E}_{I}$ if $s \in \mathcal{S}_{N}^{o f}\left(\mathcal{E}_{I}, W_{\epsilon}\right)$.

Remark 4 If $\mathbf{A} 2$ holds, then $A_{L} \mathcal{E}_{I} \oplus(W \oplus L(-H)) \subseteq \mathcal{E}_{I}$ and $\mathcal{E}_{\infty} \subseteq \mathcal{E}_{I}$. Such a set can be calculated in a variety of ways using standard techniques (cf. Remark 1), or, more usefully, as an invariant outer approximation to the mRPI set using results from [35]. In both cases, the resulting set is polytopic when all of the relevant constraints and uncertainty sets are polytopic, and the set $\mathcal{E}_{I}$ can be characterized by a finite number of linear inequalities, though an explicit representation of the set $\mathcal{E}_{I}$ is not required (cf. Remark 2 and the results of Section 5).

In general, one should expect that the initial error set $\mathcal{E}$ will be provided as a part of the problem description, and will not be an RPI set. In such cases a variety of methods may be devised for mitigating computational complexity while preserving a large region of attraction. For example, given some initial error set $\mathcal{E}$ and disturbance set $W$, one may elect to use a time-varying controller (see (32)) over some interval, and then switch to a time-invariant controller (see (39)) once $A_{L}^{k} \mathcal{E} \oplus \mathcal{E}_{k-1} \subseteq \mathcal{E}_{I}$, where $\mathcal{E}_{I}$ satisfies A2. Such a control scheme is easily shown to satisfy the system constraints for all time if the control inputs are

$$
u(k)= \begin{cases}\mu_{N}\left(s(k), k, \mathcal{E}, W_{\epsilon}\right), & \text { if } k=0 \\ \mu_{N}\left(s(k), k, A_{L}^{k} \mathcal{E} \oplus \mathcal{E}_{k-1}, W_{\epsilon}\right), & \text { if } k \in\{1, \ldots, q\} \\ \nu_{I}\left(s(k), \mathcal{E}_{I}, W_{\epsilon}\right), & \text { if } k \in\{q+1, \ldots\}\end{cases}
$$

where the integer $q$ is chosen large enough so that $A_{L}^{q} \mathcal{E} \oplus \mathcal{E}_{q-1} \subseteq \mathcal{E}_{I} \cdot{ }^{1}$ In order to increase the region of attraction for such a scheme, it is advantageous to minimize the size of the set $\mathcal{E}_{I}$, i.e. to choose $\epsilon$ as small as computational resources allow.

\section{Computation of Feedback Control Laws}

We next demonstrate how one may actually calculate feedback policies of the form (20) for the implementation of the control law (32). We consider the particular case when the constraint sets $Z$ and $X_{f}$ and uncertainty sets $W, H$ and $\mathcal{E}$ are polytopes. In this case one can define matrices $S, T$ and $U$ and a vector $b$ of appropriate dimensions such that $\mathcal{Z}$ can be expressed as

$$
\mathcal{Z}=\{(\mathbf{s}, \mathbf{e}, \mathbf{u}) \mid S \mathbf{s}+T \mathbf{e}+U \mathbf{u} \leq b\}
$$

\footnotetext{
${ }^{1}$ This condition holds if $A_{L}^{q} \mathcal{E} \subseteq \mathcal{B}_{p}^{n}(\epsilon)$. This is trivial to test, for example, in the case where the set $\mathcal{E}$ is a hypercube and $p=\infty$.
} 
so that the set of feasible control policies can be expressed as

$$
\Pi_{N}^{o f}(s, \mathcal{E}, W)=\left\{(\mathbf{M}, \mathbf{v}) \mid \begin{array}{r}
(\mathbf{M}, \mathbf{v}) \text { satisfies }(16) \\
S \mathbf{A} s+P \mathbf{v}+\delta_{e}+\delta_{w}+\delta_{\eta} \leq b \\
\delta_{e}=\left(P \mathbf{M} Q_{e}+R_{e}\right) e \\
\delta_{w}=\left(P \mathbf{M} Q_{w}+R_{w}\right) \mathbf{w} \\
\delta_{\eta}=\left(P \mathbf{M} Q_{\eta}+R_{\eta}\right) \boldsymbol{\eta} \\
\forall e \in \mathcal{E}, \forall \mathbf{w} \in W^{N}, \forall \boldsymbol{\eta} \in H^{N}
\end{array}\right\} .
$$

Recall that $a^{T} \mathbf{y} \leq e$ for all $\mathbf{y} \in \mathcal{Y}$ if and only if $\sup \left\{a^{T} \mathbf{y} \mid \mathbf{y} \in \mathcal{Y}\right\} \leq e$, where $a$ is a vector of appropriate length, $e$ is a scalar and $\sup \left\{a^{T} \mathbf{y} \mid \mathbf{y} \in \mathcal{Y}\right\}$ is the value of the support function of the set $\mathcal{Y}$ evaluated at $a$ [23]. Hence, one can eliminate the universal quantifiers in (46) to obtain the equivalent expression

$$
\Pi_{N}^{e f}(s, \mathcal{E}, W)=\left\{(\mathbf{M}, \mathbf{v}) \mid \begin{array}{r}
(\mathbf{M}, \mathbf{v}) \text { satisfies }(16) \\
F \mathbf{A} s+P \mathbf{v}+\delta_{e}+\delta_{w}+\delta_{\eta} \leq b \\
\delta_{e}=\max _{e \in \mathcal{E}}\left(P \mathbf{M} Q_{e}+R_{e}\right) e \\
\delta_{w}=\max _{\mathbf{w} \in W^{N}}\left(P \mathbf{M} Q_{w}+R_{w}\right) \mathbf{w} \\
\delta_{\eta}=\max _{\boldsymbol{\eta} \in H^{N}}\left(P \mathbf{M} Q_{\eta}+R_{\eta}\right) \boldsymbol{\eta}
\end{array}\right\}
$$

where the matrices $P, Q_{e}, Q_{w}, Q_{\eta}, R_{e}, R_{w}$ and $R_{\eta}$ are defined in the Appendix, and the maximizations are performed row-wise. Note that all of the maxima in (47) are attained since the sets $\mathcal{E}, W$ and $H$ are assumed compact. A pair $(\mathbf{M}, \mathbf{v}) \in \Pi_{N}^{e f}(s, \mathcal{E})$ can thus be found by forming the dual optimization problem associated with each element of the vectors $\delta_{e}, \delta_{w}$ and $\delta_{\eta}$ and introducing slack variables to form a single linear program, whose size is polynomial in the number of constraints defining the sets $\mathcal{Z}, \mathcal{E}, W$ and $H$. To show this, suppose that the sets $W^{N}, H^{N}$ and $\mathcal{E}$ are defined as $W^{N}:=\left\{\mathbf{w} \mid G_{w} \mathbf{w} \leq h_{w}\right\}$, $H^{N}:=\left\{\boldsymbol{\eta} \mid G_{\eta} \boldsymbol{\eta} \leq h_{\eta}\right\}$ and $\mathcal{E}:=\left\{e \mid G_{e} \boldsymbol{\eta} \leq h_{e}\right\}$, respectively, where the matrices $G_{w}, G_{\eta}$ and $G_{e}$ and vectors $h_{w}, h_{\eta}$ and $h_{e}$ are of appropriate dimension. Following the dualization procedure that can be found, for example, in $[6,20],(47)$ can be rewritten as:

$$
\Pi_{N}^{e f}(s, \mathcal{E}, W)=\left\{\begin{array}{l|r} 
& \begin{array}{r}
(\mathbf{M}, \mathbf{v}) \text { satisfies }(16), \exists\left(\mathbf{Z}_{w}, \mathbf{Z}_{e}, \mathbf{Z}_{\eta}\right) \\
F \mathbf{A} s+P \mathbf{v}+\delta_{e}+\delta_{w}+\delta_{\eta} \leq b \\
\mathbf{Z}_{e} G_{e}=P \mathbf{M} Q_{e}+R_{e}, \quad \delta_{e}=\mathbf{Z}_{e} h_{e} \\
\mathbf{Z}_{w} G_{w}=P \mathbf{M} Q_{w}+R_{w}, \delta_{w}=\mathbf{Z}_{w} h_{w} \\
\mathbf{Z}_{\eta} G_{\eta}=P \mathbf{M} Q_{\eta}+R_{\eta}, \delta_{\eta}=\mathbf{Z}_{\eta} h_{\eta} \\
\left(\mathbf{Z}_{e}, \mathbf{Z}_{w}, \mathbf{Z}_{\eta}\right) \geq 0
\end{array}
\end{array}\right\}
$$

where all inequalities are element-wise. Computation of a feasible policy in this set is thus possible via the solution of a single linear program with a number of variables and constraints that is a quadratic function of the horizon length $N$ and the number of inequalities that characterize the sets $\mathcal{Z}, W, H$ and $\mathcal{E}$.

In particular, it is important to note that it is not necessary to explicitly perform the Minkowski summation of error sets in the calculation of the time varying control law (32), since only the support functions of these sets is of interest. Given an initial error set $\mathcal{E}$ at time 0 , one needs to calculate, at each time $k$, a feasible policy pair $(\mathbf{M}, \mathbf{v}) \in \Pi_{N}^{e f}\left(s(k), A_{L}^{k} \mathcal{E} \oplus \mathcal{E}_{k-1}\right)$. In this case the vector $\delta_{e}$ in (47) can be 
written as

$$
\begin{aligned}
\delta_{e} & =\max _{e \in A_{L}^{k} \mathcal{E} \oplus \mathcal{E}_{k-1}}\left(P \mathbf{M} Q_{e}+R_{e}\right) e \\
& =\max _{e \in \mathcal{E}}\left(P \mathbf{M} Q_{e}+R_{e}\right) A_{L}^{k} e+\max _{e \in \mathcal{E}_{k-1}}\left(P \mathbf{M} Q_{e}+R_{e}\right) e \\
& =\max _{e \in \mathcal{E}}\left(P \mathbf{M} Q_{e}+R_{e}\right) A_{L}^{k} e+\sum_{i=0}^{k-1}\left[\left(\max _{w \in W}\left(P \mathbf{M} Q_{e}+R_{e}\right) A_{L}^{i} w\right)+\left(\max _{\eta \in H}\left(P \mathbf{M} Q_{e}+R_{e}\right) A_{L}^{i}(-L) \eta\right)\right],
\end{aligned}
$$

and one may dualize each row of each component of this summation, forming a single linear program whose size increases polynomially with time $k$.

An identical procedure may be used in the computation of an element of the set $\Pi_{N}^{e f}\left(s, \mathcal{E}_{I}, W\right)$ in the implementation of the time-invariant control law (39) (cf. Remark 3), resulting in a tractable LP of fixed and finite complexity, where once again it is not necessary to explicitly form the Minkowski sum in (38), and where the support function of $\mathcal{E}_{I}$ can be determined using an implicit representation of a Minkowski sum of a finite number of polytopes as in [35]. In particular, the results in [35] demonstrate that, for any $p$-norm and approximation accuracy $\epsilon>0$, there exists a finite integer $r$ and a scalar $\beta>1$, such that $\mathcal{E}_{\infty} \subseteq \mathcal{E}_{I} \subseteq \mathcal{E}_{\infty} \oplus \mathcal{B}_{p}^{n}(\epsilon)$, where

$$
\mathcal{E}_{I}=\beta \bigoplus_{i=0}^{r} A_{L}^{i}(W \oplus L(-H))
$$

It is of particular importance to note that the number of terms $r$ in this summation is primarily dependent on the spectral radius of the matrix $(A-L C)$, which is selected by the designer of the controller [34]. One can thus write

$$
\delta_{e}=\beta \sum_{i=0}^{r}\left[\left(\max _{w \in W}\left(P \mathbf{M} Q_{e}+R_{e}\right) A_{L}^{i} w\right)+\left(\max _{\eta \in H}\left(P \mathbf{M} Q_{e}+R_{e}\right) A_{L}^{i}(-L) \eta\right)\right]
$$

and one again dualize each row of this summation to obtain a representation of the set $\Pi_{N}^{e f}\left(s, \mathcal{E}_{I}, W\right)$ as in (48) in a number of variables and constraints which is polynomial in the size of the problem data.

\section{$5.1 \quad$ Numerical Example}

We consider the discrete-time system

$$
\begin{aligned}
x^{+} & =\left[\begin{array}{ll}
1 & 1 \\
0 & 1
\end{array}\right] x+\left[\begin{array}{c}
0.2 \\
1
\end{array}\right] u+w \\
y & =\left[\begin{array}{ll}
1 & 1
\end{array}\right] x+\eta
\end{aligned}
$$


with stable feedback gain $K$ and observer gain $L$ chosen as $K=\left[\begin{array}{ll}-0.75 & -1.85\end{array}\right]$ and $L=\left[\begin{array}{ll}1.15 & 0.65\end{array}\right]^{\prime}$. The sets $\mathcal{Z}, \mathcal{E}, W$ and $H$ are defined as

$$
\begin{aligned}
Z & :=\left\{(x, u) \in \mathbb{R}^{2} \times \mathbb{R} \mid \begin{array}{r}
-3 \leq x_{1} \leq 25 \\
-3 \leq x_{2} \leq 25 \\
|u| \leq 5
\end{array}\right\} \\
\mathcal{E} & :=\left\{e \in \mathbb{R}^{2} \mid\|e\|_{\infty} \leq 0.4\right\} \\
W & :=\left\{w \in \mathbb{R}^{2} \mid\|w\|_{\infty} \leq 0.1\right\} \\
H & :=\left\{\eta \in \mathbb{R}^{2} \mid\|\eta\|_{\infty} \leq 0.1\right\}
\end{aligned}
$$

where $x_{i}$ is the $i^{\text {th }}$ element of $x$. In order to obtain the set $X_{f}$, we calculate the maximal RPI set compatible with $Z$ for the system (25) using the method of [23, Alg. 6.2]. We consider the set of feasible initial state estimates $\mathcal{S}_{i}^{o f}(\mathcal{E}, W)$ (equivalently $\mathcal{S}_{i}^{e f}(\mathcal{E}, W)$ ) for this system. For comparison, we also consider the sets $S_{i}^{K}(\mathcal{E}, W)$ for which a feasible control policy can be found when the policy is parameterized in terms of perturbations to a fixed state feedback gain, such that $u_{j}=K s_{j}+c_{j}$. Recall that $S_{i}^{K}(\mathcal{E}, W) \subseteq S_{i}^{o f}(\mathcal{E}, W)$ for all $i \in\{0,1, \ldots\}$ (cf. Remark 1). The resulting sets of feasible initial state estimates for this system are shown in Figure 1.

\section{Conclusions}

The main contribution of this paper is to propose a new class of time-invariant receding horizon output feedback control laws for control of linear systems subject to bounded disturbances that guarantee robust constraint satisfaction for the resulting closed-loop system for all time. The proposed method is based on a fixed linear state observer combined with optimization over the class of feedback policies which are affine in the sequence of prior outputs; this problem is non-convex, but can be convexified using an appropriate reparameterization. As a consequence, receding horizon control laws in the proposed class can be computed using standard techniques in convex optimization, while providing a larger region of attraction than methods based on calculating control perturbations to a static linear feedback law.

We have only considered the problem of finding a feasible control policy at each time, without regard to optimality. It is possible to define a variety of cost functions to motivate the selection from amongst this feasible set of policies, and we have not addressed any stability results which may be derived based on this selection; see, however, $[19,20,22]$ for related results in the state feedback case.

\section{Appendix A: Matrix Definitions}

Define $\mathfrak{A} \in \mathbb{R}^{n(N+1) \times n}$ and $\mathfrak{E} \in \mathbb{R}^{n(N+1) \times n N}$ as

$$
\mathfrak{A}(L):=\left[\begin{array}{c}
I_{n} \\
(A-L C) \\
(A-L C)^{2} \\
\vdots \\
(A-L C)^{N}
\end{array}\right], \quad \mathfrak{E}(L):=\left[\begin{array}{cccc}
0 & 0 & \cdots & 0 \\
I_{n} & 0 & \cdots & 0 \\
(A-L C) & I_{n} & \cdots & 0 \\
\vdots & \vdots & \ddots & \vdots \\
(A-L C)^{N-1} & (A-L C)^{N-2} & \cdots & I_{n}
\end{array}\right]
$$

so that $\mathbf{A}:=\mathfrak{A}(0), \mathbf{E}:=\mathfrak{E}(0), \Phi:=\mathfrak{A}(L)$ and $\Gamma:=\mathfrak{E}(L)$ The matrices $\mathcal{B} \in \mathbb{R}^{n N \times m N}, \mathcal{L} \in \mathbb{R}^{n N \times q N}$, $\mathbf{B} \in \mathbb{R}^{n(N+1) \times m N}$, and $\mathbf{C} \in \mathbb{R}^{q N \times n(N+1)}$ are defined as $\mathcal{B}:=\left(I_{N} \otimes B\right), \mathcal{L}:=\left(I_{N} \otimes L\right), \mathbf{B}:=\mathbf{E} \mathcal{B}$ and $\mathbf{C}:=\left[\left(I_{N} \otimes C\right) 0\right]$ respectively. 


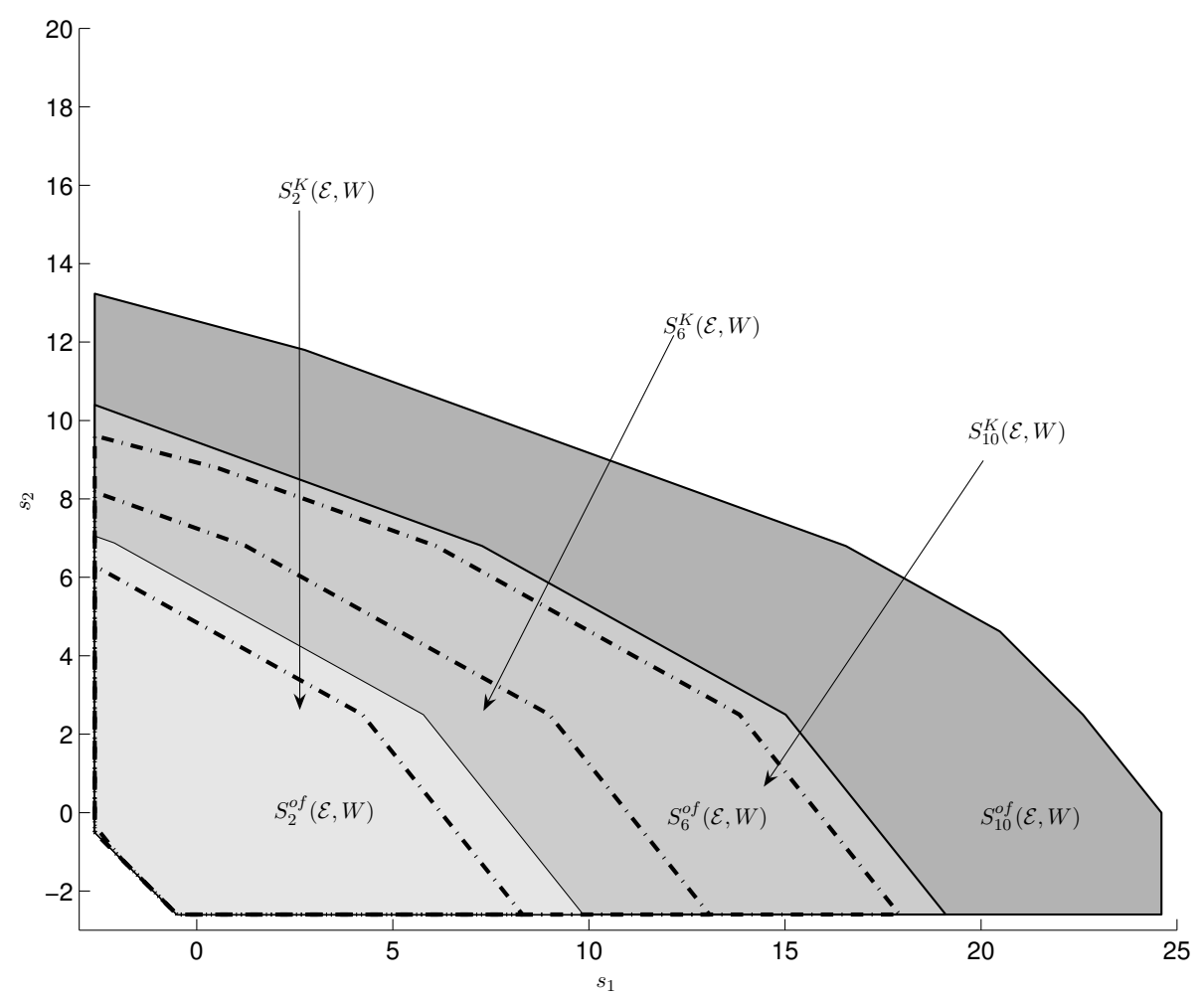

Figure 1. Feasible initial state estimate sets $\mathcal{S}_{i}^{o f}(\mathcal{E}, W)$ and $\mathcal{S}_{i}^{K}(\mathcal{E}, W)$ for $i \in\{2,6,10\}$

If the constraint set $\mathcal{Z}$ is polytopic and defined as in (45), then we define the matrices in (47) as $P:=(S \mathbf{B}+U), Q_{e}:=\mathbf{C} \Phi, R_{e}:=(S \mathbf{E} \mathcal{L} \mathbf{C}+T) \Phi, Q_{w}:=\mathbf{C} \Gamma, R_{w}:=(S \mathbf{E} \mathcal{L} \mathbf{C}+T) \Gamma, Q_{\eta}:=(I-\mathbf{C} \Gamma \mathcal{L})$ and $R_{\eta}:=(S \mathbf{E}(I-\mathcal{L} \mathbf{C} \Gamma)-T \Gamma) \mathcal{L}$.

A bit of algebra confirms that the matrix identities $\mathbf{E}=(I+\mathbf{E} \mathcal{L} \mathbf{C}) \Gamma$ and $\mathbf{A}=(I+\mathbf{E} \mathcal{L} \mathbf{C}) \Phi$ hold, so that one may also use the equivalent matrix definitions $R_{e}:=S \mathbf{A}-(S-T) \Phi, R_{w}:=S \mathbf{E}-(S-T) \Gamma$ and $R_{\eta}:=(S-T) \Gamma \mathcal{L}$ above.

\section{References}

[1] J. S. Baras and N. S. Patel. Robust control of set-valued discrete-time dynamical systems. IEEE Transactions on Automatic Control, 43(1):61-75, January 1998.

[2] A. Bemporad. Reducing conservativeness in predictive control of constrained systems with disturbances. In Proc. 37th IEEE Conference on Decision and Control, pages 1384-1391, Tampa, FL, USA, December 1998.

[3] A. Bemporad and A. Garulli. Output-feedback predictive control of constrained linear systems via set-membership state estimation. International Journal of Control, 73(8):655-665, May 2000.

[4] A. Bemporad and M. Morari. Robust Model Predictive Control: A Survey in Robustness in Identifica- 
tion and Control, volume 245 of Lecture Notes in Control and Information Sciences, pages 207-226. Ed. A. Garulli, A. Tesi, and A. Vicino. Springer-Verlag, 1999.

[5] A. Ben-Tal, S. Boyd, and A. Nemirovski. Extending scope of robust optimization: Comprehensive robust counterparts of uncertain problems. Mathematical Programming, 107(1-2):63-89, June 2006.

[6] A. Ben-Tal, A. Goryashko, E. Guslitzer, and A Nemirovski. Adjustable robust solutions of uncertain linear programs. Mathematical Programming, 99(2):351-376, March 2004.

[7] D. P. Bertsekas. Dynamic Programming and Optimal Control, volume 1. Athena Scientific, 2nd edition, 2000.

[8] D. P. Bertsekas and I. B. Rhodes. On the minimax reachability of target sets and target tubes. Automatica, 7(2):233-247, 1971.

[9] D. P. Bertsekas and I. B. Rhodes. Recursive state estimation for a set-membership description of uncertainty. IEEE Transactions on Automatic Control, 16(2):117-128, April 1971.

[10] D. P. Bertsekas and I. B. Rhodes. Sufficiently informative functions and the minimax feedback control of uncertain dynamic systems. IEEE Transactions on Automatic Control, AC-18(2):117-124, April 1973.

[11] F. Blanchini. Set invariance in control. Automatica, 35(1):1747-1767, November 1999.

[12] S. P. Boyd and C. H. Barratt. Linear Controller Design: Limits of Performance. Prentice Hall, 1991.

[13] E. F. Camacho and C. Bordons. Model Predictive Control. Springer, second edition, 2004.

[14] L. Chisci, J. A. Rossiter, and G. Zappa. Systems with persistent state disturbances: predictive control with restricted constraints. Automatica, 37(7):1019-1028, July 2001.

[15] L. Chisci and G. Zappa. Feasibility in predictive control of constrained linear systems: the output feedback case. International Journal of Robust and Nonlinear Control, 12:465-487, April 2002.

[16] M. A. Dahleh and I. J. Diaz-Bobillo. Control of Uncertain Systems. Prentice Hall, 1995.

[17] R. Findeisen, L. Imsland, Frank Allgöwer, and B. A. Foss. State and output feedback nonlinear model predictive control: An overview. European Journal of Control, 9(2-3):190-207, 2003.

[18] E. G. Gilbert and I. Kolmanovsky. Fast reference governors for systems with state and control constraints and disturbance inputs. International Journal of Robust and Nonlinear Control, 9(15):11171141, 1999.

[19] P. J. Goulart and E. C. Kerrigan. On a class of robust receding horizon control laws for constrained systems. Provisionally accepted to Automatica. Available as Technical Report CUED/F-INFENG/TR.532, Cambridge University Engineering Department, August 2005.

[20] P. J. Goulart, E. C. Kerrigan, and J. M. Maciejowski. Optimization over state feedback policies for robust control with constraints. Automatica, 42(4):523-533, April 2006.

[21] E. Guslitser. Uncertainty-immunized solutions in linear programming. Master's thesis, Technion, Israeli Institute of Technology, June 2002.

[22] E. C. Kerrigan and T. Alamo. A convex parameterization for solving constrained min-max problems with a quadratic cost. In Proc. 2004 American Control Conference, Boston, MA, USA, June 2004.

[23] I. Kolmanovsky and E. G. Gilbert. Theory and computations of disturbance invariant sets for discretetime linear systems. Mathematical Problems in Engineering, 4(4):317-363, 1998.

[24] W. I. Langson, I. Chryssochoos, S. V. Raković, and D. Q. Mayne. Robust model predictive control using tubes. Automatica, 40:125-133, 2004.

[25] Y. I. Lee and B. Kouvaritakis. Constrained receding horizon predictive control for systems with disturbances. International Journal of Control, 72(11):1027-1032, August 1999.

[26] Y. I. Lee and B. Kouvaritakis. Receding-horizon output feedback control for systems with input saturation. IEE Proceedings on Control Theory and Applications, 148:109-115, 2001.

[27] J. Löfberg. Approximations of closed-loop MPC. In Proc. 42nd IEEE Conference on Decision and Control, pages 1438-1442, Maui, Hawaii, USA, December 2003.

[28] J. M. Maciejowski. Predictive Control with Constraints. Prentice Hall, UK, 2002.

[29] D. Q. Mayne, S. V. Raković, R. Findeisen, and F. Allgöwer. Robust output feedback model predictive control of constrained linear systems. Submitted to Automatica, August 2005.

[30] D. Q. Mayne, J. B. Rawlings, C. V. Rao, and P. O. M. Scokaert. Constrained model predictive control: Stability and optimality. Automatica, 36(6):789-814, June 2000. Survey paper. 
[31] D. Q. Mayne, M. M. Seron, and S. V. Raković. Robust model predictive control of constrained linear systems with bounded disturbances. Automatica, 41(2):219-24, February 2005.

[32] T. Perez, H. Haimovich, and G. C. Goodwin. On optimal control of constrained linear systems with imperfect state information and stochastic disturbances. International Journal of Robust and Nonlinear Control, 14(4):379-393, April 2004.

[33] S. J. Qin and T. A. Badgwell. A survey of industrial model predictive control technology. Control Engineering Practice, 11:733-764, 2003.

[34] S. V. Raković, E. C. Kerrigan, K. I. Kouramas, and D. Q. Mayne. Invariant approximations of robustly positively invariant sets for constrained linear discrete-time systems subject to bounded disturbances. Technical Report CUED/F-INFENG/TR.473, Cambridge University Engineering Department, January 2004.

[35] S. V. Raković, E. C. Kerrigan, K. I. Kouramas, and D. Q. Mayne. Invariant approximations of the minimal robust positively invariant set. IEEE Transactions on Automatic Control, 50(3), March 2005.

[36] S. V. Raković and D. Q. Mayne. Regulation of discrete-time linear systems with positive state and control constraints and bounded disturbances. In Proc. 16th IFAC World Congress on Automatic Control, Prague, Czech Republic, July 2005.

[37] A. Richards and J. How. Robust model predictive control with imperfect information. In Proc. 2005 American Control Conference, pages 268-274, Portland, OR, USA, June 2005.

[38] F. Schweppe. Recursive state estimation: Unknown but bounded errors and system inputs. IEEE Transactions on Automatic Control, 13(1):22-28, February 1968.

[39] P. O. M. Scokaert and D. Q. Mayne. Min-max feedback model predictive control for constrained linear systems. IEEE Transactions on Automatic Control, 43(8):1136-1142, August 1998.

[40] J. S. Shamma and K. T. Tu. Set valued observers and optimal disturbance rejection. IEEE Transactions on Automatic Control, 44(2):253-264, February 1999.

[41] J. S. Shamma and K. Y. Tu. Output feedback control for systems with constraints and saturations: scalar control case. Systems 85 Control Letters, 35(1):1-11, August 1998.

[42] M. Sznaier and J. Bu. Mixed $l_{1} / \mathcal{H}_{\infty}$ control of MIMO systems via convex optimization. IEEE Transactions on Automatic Control, 43(9):1229-1241, September 1998.

[43] D. H. van Hessem and O. Bosgra. Stochastic closed-loop model predictive control of continuous nonlinear chemical processes. Journal of Process Control, 16(3):225-241, March 2005.

[44] Y. W. Wang and J. B. Rawlings. A new robust model predictive control method II: Theory and computation. Journal of Process Control, 14(3):231-247, April 2004.

[45] H. S. Witsenhausen. A minimax control problem for sampled linear systems. IEEE Transactions on Automatic Control, AC-13(1):5-21, 1968.

[46] J. Yan and R. R. Bitmead. Incorporating state estimation into model predictive control and its application to network traffic control. Automatica, 41(4):595-604, April 2005. 